\title{
AN EXPLICIT FORMULA OF CAUCHY-SZEGÖ KERNEL FOR QUATERNIONIC SIEGEL UPPER HALF SPACE AND APPLICATIONS
}

\author{
DER-CHEN CHANG, XUAN THINH DUONG, JI LI, WEI WANG AND QINGYAN WU
}

\begin{abstract}
In this paper we obtain an explicit formula of Cauchy-Szegö kernel for quaternionic Siegel upper half space, and then based on this, we prove that the Cauchy-Szegö projection on quaternionic Heisenberg group is a Calderón-Zygmund operator via verifying the size and regularity conditions for the kernel. Next, we also obtain a suitable version of pointwise lower bound for the kernel, which further implies the characterisations of the boundedness and compactness of commutator of the Cauchy-Szegö operator via the BMO and VMO spaces on quaternionic Heisenberg group, respectively.
\end{abstract}

\section{IntRoduCtion AND STATEMENT OF MAIN RESUltS}

1.1. Background. The quaternion numbers $x=x_{1}+x_{2} \mathbf{i}+x_{3} \mathbf{j}+x_{4} \mathbf{k}$ with $\mathbf{i}^{2}=\mathbf{j}^{2}=\mathbf{k}^{2}=$ $\mathbf{i j k}=-1$ were introduced by Hamilton as an extension of complex numbers in the $19^{t h}$ century and applied to mechanics in three-dimensional space. A feature of quaternions is that multiplication of two quaternions is noncommutative. Let $\mathbb{H}$ be the system of quaternion numbers and let $\operatorname{Re} x$ and $\operatorname{Im} x$ denote the real part and imaginary part of $x$ respectively. Then $\operatorname{Re} x=x_{1}$ and $\operatorname{Im} x=x_{2} \mathbf{i}+x_{3} \mathbf{j}+x_{4} \mathbf{k}$. The $n$-dimensional quaternion space $\mathbb{H}^{n}$ is the collection of $n$-tuples $\left(q_{1}, \cdots, q_{n}\right)$. An $\mathbb{H}$-valued function $f: \Omega \rightarrow \mathbb{H}$ is called regular if $\bar{\partial} f=0$, where

$$
\bar{\partial}=\frac{\partial}{\partial x_{1}}+\sum_{m=1}^{3} \mathbf{i}_{m} \frac{\partial}{\partial x_{m+1}} \text { or } \bar{\partial}=\frac{\partial}{\partial x_{1}}+\sum_{m=1}^{3} \frac{\partial}{\partial x_{m+1}} \mathbf{i}_{m}, \quad \mathbf{i}_{1}=\mathbf{i}, \quad \mathbf{i}_{2}=\mathbf{j}, \quad \mathbf{i}_{3}=\mathbf{k} .
$$

The theory of regular functions of several quaternionic variables began in 1980s. So far there are several fundamental results established for the quaternionic counterpart of the theory of several complex variables, e.g., Hartogs phenomenon, $k$-Cauchy-Fueter complexes, quaternionic pluripotential theory etc (see for example [1, 9, 33, 35] and the references therein). Because of the non-commutativity of the quaternion $\mathbb{H}$, many new phenomena emerge, e.g. the product of two regular functions is not regular in general. Hence, proofs of almost all results are completely different from the standard setting of complex variables.

Parallel to the setting of several complex variables, people are also interested in the Hardy space of regular functions over a bounded domain in $\mathbb{H}^{n}$, in particular, over the unit ball. By quaternionic Cayley transformation, it is equivalent to considering the Hardy space over the Siegel upper half domain

$$
\mathcal{U}_{n}:=\left\{q=\left(q_{1}, \cdots, q_{n}\right)=\left.\left(q_{1}, q^{\prime}\right) \in \mathbb{H}^{n}\left|\operatorname{Re} q_{1}>\right| q^{\prime}\right|^{2}\right\},
$$

where $q^{\prime}=\left(q_{2}, \cdots, q_{n}\right) \in \mathbb{H}^{n-1}$, whose boundary $\partial \mathcal{U}_{n}:=\left\{\left.\left(q_{1}, q^{\prime}\right) \in \mathbb{H}^{n}\left|\operatorname{Re} q_{1}=\right| q^{\prime}\right|^{2}\right\}$ is a quadratic hypersurface, which can be identified with the quaternionic Heisenberg group $\mathscr{H}^{n-1}$. For the notation and details of definition we refer to Section 2.

Date: September 4, 2019.

2010 Mathematics Subject Classification. 32A25, 32A26, 43A80, 42B20.

Key words and phrases. Cauchy-Szegö kernel, quaternionic Siegel upper half space, Calderón-Zygmund kernel. 
We point out that the quaternionic Heisenberg group $\mathscr{H}^{n-1}$ plays the fundamental role in quaternionic analysis and geometry [7, 20, 34, 28, Its analytic and geometric behaviours are different from the usual Heisenberg group in many aspects, e.g., there does not exist nontrivial quasiconformal mapping between the quaternionic Heisenberg group 27] while quasiconformal mappings between Heisenberg groups are abundant [21, 22.

The identification $\partial \mathcal{U}_{n}$ with the quaternionic Heisenberg group $\mathscr{H}^{n-1}$ via the projection (2.3) helps us to determine the kernel of the Cauchy-Szegö projection from $L^{2}\left(\partial \mathcal{U}_{n}\right)$ to $H^{2}\left(\partial \mathcal{U}_{n}\right)[3$. This was just obtained recently by the first, fourth authors and Markina [4]. To be more explicit, we recall the result as follows.

Theorem A (4]). The Cauchy-Szegö kernel is given by

$$
S(q, p)=s\left(q_{1}+\bar{p}_{1}-2 \sum_{k=2}^{n} \bar{p}_{k} q_{k}\right),
$$

for $p=\left(p_{1}, p^{\prime}\right)=\left(p_{1}, \cdots, p_{n}\right) \in \mathcal{U}_{n}, q=\left(q_{1}, q^{\prime}\right)=\left(q_{1}, \cdots, q_{n}\right) \in \mathcal{U}_{n}$, where

$$
s(\sigma)=c_{n-1} \frac{\partial^{2(n-1)}}{\partial x_{1}^{2(n-1)}} \frac{\bar{\sigma}}{|\sigma|^{4}}, \quad \sigma=x_{1}+x_{2} \mathbf{i}+x_{3} \mathbf{j}+x_{4} \mathbf{k} \in \mathbb{H},
$$

with the real constant $c_{n-1}$ depending only on $n$. The Cauchy-Szegö kernel satisfies the reproducing property in the following sense $F(q)=\int_{\partial \mathcal{U}_{n}} S(q, \xi) F^{b}(\xi) d \beta(\xi), q \in \mathcal{U}_{n}$, whenever $F \in \mathcal{H}^{2}\left(\mathcal{U}_{n}\right)$ and $F^{b}$ its boundary value on $\partial \mathcal{U}_{n}$.

Recall that the standard Cauchy-Szegö projection on the usual Heisenberg group has an explicit formula, which, together with the appropriate quasi-metric and the volume function, implies that the Cauchy-Szegö kernel on the Heisenberg group is a Calderón-Zygmund kernel (we refer to [30, Section 2, Chapter XII] for background and details). Hence, Stein ([30, Theorem 2, Chapter XII]) summarised and stated the main conclusion as:

"The Cauchy-Szegö projection on the Heisenberg group has an extension to a bounded operator on $L^{p}$ for all $1<p<\infty$."

Moreover, it is also known that from the explicit formula of the Cauchy-Szegö kernel, especially the pointwise lower bound, one can obtain the characterisation of the boundedness and compactness of the commutator of the Cauchy-Szegö projection via the BMO and VMO spaces, respectively. See the recent results in [11, Section 7.2] and [5], respectively.

It is natural to ask, whether the Cauchy-Szegö kernel on the quaternionic Heisenberg group determined in Theorem A above has an explicit formula and a Calderón-Zygmund theory parallel to [30, Section 2, Chapter XII]?

1.2. Statement of main results. The aim of this paper is to address this question, providing an explicit formula of the Cauchy-Szegö kernel for the quaternion Siegel upper half-space. Then by using this formula we verify that it is a standard Calderón-Zygmund kernel with respect to the quasi-metric $\rho$ (defined in Section 2, which is a standard definition in general stratified Lie group, see for example [10, Chapter 1]), that is, it satisfies the standard size, and smoothness conditions in terms of $\rho$. Moreover, we also provide a suitable version of pointwise kernel lower bound from the explicit formula of the kernel, which was motivated by recent works on commutator estimates such as [19, 11, 14,.

As a direct application, we establish the characterisation of the BMO space via the commutator $[b, \mathcal{C}]$, which is parallel to the classical setting in [8] for the Riesz transforms on $\mathbb{R}^{n}$, and some closely related recent results in [18] for two weight commutators, 25] for the Cauchy integral along Lipschitz curves, 14] for the Riesz transform on stratified Lie groups, [23, 24, 16] for singular integrals with rough kernels, [19] for the $L^{p}$ to $L^{q}$ boundedness of commutators with applications to the Jacobian operator, 13 for the Cauchy integrals on 
certain pseudoconvex domains in $\mathbb{C}^{n}$ with minimal regularity condition on the boundary. See also the general frame on space of homogeneous type in [11, which restructured the proof of 15, 12, 26 and the references therein. Note that the weakest sufficient condition known so far on the kernel lower bound of Calderón-Zygmund operators which gives the characterisation of the BMO via commutator was due to Hytönen [19.

The characterisation of the compactness of the commutator $[b, \mathcal{C}]$ is also studied by using the pointwise kernel lower bound that we obtained. For the previous related result on compactness of commutators, we refer to (but not restricted to) [32, 5, 17, 31, 13.

We now state our first main result.

Theorem 1.1. The explicit formula of Cauchy-Szegö kernel for the quaternionic Siegel upper half-space $\mathcal{U}_{n}$ is given by

$$
S(q, p)=s\left(q_{1}+\bar{p}_{1}-2 \sum_{k=2}^{n} \bar{p}_{k} q_{k}\right)
$$

for $p=\left(p_{1}, p^{\prime}\right)=\left(p_{1}, \cdots, p_{n}\right) \in \mathcal{U}_{n}, q=\left(q_{1}, q^{\prime}\right)=\left(q_{1}, \cdots, q_{n}\right) \in \mathcal{U}_{n}$, where

$$
\begin{aligned}
s(\sigma)= & c_{n-1} \frac{4(2 n-2) !}{|z|^{4}(z-\bar{z})^{3}} \mathbf{i} \\
& \times\left\{\operatorname{Im}\left[\frac{\bar{z}^{2}}{z^{2 n-2}}\left(z+(2 n-1) \frac{z-\bar{z}}{2}\right)\right] \bar{\sigma}-\operatorname{Im}\left[\frac{\bar{z}^{2}}{z^{2 n-3}}\left(z+(2 n-2) \frac{z-\bar{z}}{2}\right)\right]\right\},
\end{aligned}
$$

here $\sigma=x_{1}+x_{2} \mathbf{i}+x_{3} \mathbf{j}+x_{4} \mathbf{k} \in \mathbb{H}, z=x_{1}+|\operatorname{Im} \sigma| \mathbf{i}$, and $c_{n-1}$ is the one in Theorem $A$.

For any function $F: \mathcal{U}_{n} \rightarrow \mathbb{H}$, we write $F_{\varepsilon}$ for its "vertical translate", where the vertical direction is given by the positive direction of $\operatorname{Re} q_{1}: F_{\varepsilon}(q)=F(q+\varepsilon \mathbf{e})$, where $\mathbf{e}=(1,0,0, \cdots, 0) \in \mathbb{H}^{n}$. If $\varepsilon>0$, then $F_{\varepsilon}$ is defined in a neighborhood of $\partial \mathcal{U}_{n}$. In particular, $F_{\varepsilon}$ is defined on $\partial \mathcal{U}_{n}$.

The Cauchy-Szegö projection operator $\mathcal{C}$ can be defined via the "vertical translate" from Cauchy-Szegö kernel for $\mathcal{U}_{n}$ by

$$
(\mathcal{C} f)(q)=\lim _{\varepsilon \rightarrow 0} \int_{\partial \mathcal{U}_{n}} S(q+\varepsilon \mathbf{e}, p) f(p) d \beta(p), \quad \forall f \in L^{2}\left(\partial \mathcal{U}_{n}\right), \quad q \in \partial \mathcal{U}_{n},
$$

where the limit exists in the $L^{2}\left(\partial \mathcal{U}_{n}\right)$ norm and $\mathcal{C}(f)$ is the boundary limit of some function in $\mathcal{H}^{2}\left(\mathcal{U}_{n}\right)$.

In view of the action of the quaternionic Heisenberg group, the operator $\mathcal{C}$ can be explicitly described as a convolution operator on this group:

$$
(\mathcal{C} f)(g)=(f * K)(g)=p \cdot v \cdot \int_{\mathscr{H}^{n-1}} K\left(h^{-1} \cdot g\right) f(h) d h,
$$

where the kernel $K(g)$ is defined in Section 2 below. We can write

$$
(\mathcal{C} f)(g)=p \cdot v \cdot \int_{\mathscr{H}^{n-1}} K(g, h) f(h) d h,
$$

where $K(g, h)=K\left(h^{-1} \cdot g\right)$ for $g \neq h$. Note that (1.5) holds whenever $f$ is an $L^{2}$ function supported in a compact set, for ever $g$ outside the support of $f$.

As the second main result, we now prove the size and regularity estimate for the CauchySzegö kernel as follows.

Theorem 1.2. Suppose $j=1, \ldots, 4 n-4$, and we denote $Y_{j}$ the left-invariant vector fields on $\mathscr{H}^{n-1}$ (defined as in (2.5) in Section 2). Then we have

$$
\left|Y_{j} K(g)\right| \lesssim \frac{1}{\rho(g, \mathbf{0})^{Q+1}}, \quad g \in \mathscr{H}^{n-1} \backslash\{\mathbf{0}\},
$$


where $\mathbf{0}$ is the neutral element of $\mathscr{H}^{n-1}$.

Then we further have the Cauchy-Szegö kernel $K(g, h)$ on $\mathscr{H}^{n-1}(g \neq h)$ satisfies the following conditions.

(i) $|K(g, h)| \lesssim \frac{1}{\rho(g, h)^{Q}} ;$

(ii) $\left|K(g, h)-K\left(g_{0}, h\right)\right| \lesssim \frac{\rho\left(g, g_{0}\right)}{\rho\left(g_{0}, h\right)^{Q+1}}, \quad$ if $\rho\left(g_{0}, h\right) \geq c \rho\left(g, g_{0}\right)$;

(iii) $\left|K(g, h)-K\left(g, h_{0}\right)\right| \lesssim \frac{\rho\left(h, h_{0}\right)}{\rho\left(g, h_{0}\right)^{Q+1}}, \quad$ if $\rho\left(g, h_{0}\right) \geq c \rho\left(h, h_{0}\right)$

for some constant $c>0$, where $Q=4 n+2$ is the homogeneous dimension of $\mathscr{H}^{n-1}$ and $\rho$ is defined in Section 2.

As a consequence, we see that the Cauchy-Szegö projection $\mathcal{C}$ is a standard CalderónZygmund operator, falling into the scope of the framework set forth in Chapter 1, Section 6.5 in 30. Therefore, Theorem 3 in Chapter 1 in 30 ( $L^{p}$ boundedness, $\left.1<p<\infty\right)$ is applicable to $\mathcal{C}$. Moreover, concerning the Hardy, BMO and VMO spaces studied in 10] on general homogeneous groups, we have the endpoint estimate for $\mathcal{C}$. And from [8, 32, we also have the boundedness and compactness of the commutator of $\mathcal{C}$. We formulate these results as follows.

Corollary 1.3. Let all the notation be the same as above.

(1) $\mathcal{C}$ extends to a bounded operator on $L^{p}\left(\mathscr{H}^{n-1}\right)$ for $1<p<\infty$;

(2) $\mathcal{C}$ is of weak type $(1,1)$;

(3) $\mathcal{C}$ is bounded from $H^{1}\left(\mathscr{H}^{n-1}\right) \rightarrow L^{1}\left(\mathscr{H}^{n-1}\right)$;

(4) $\mathcal{C}$ is bounded from $L^{\infty}\left(\mathscr{H}^{n-1}\right) \rightarrow \operatorname{BMO}\left(\mathscr{H}^{n-1}\right)$;

(5) $[b, \mathcal{C}]$ is bounded on $L^{p}\left(\mathscr{H}^{n-1}\right)$ for $1<p<\infty$ if $b \in \operatorname{BMO}\left(\mathscr{H}^{n-1}\right)$;

(6) $[b, \mathcal{C}]$ is compact on $L^{p}\left(\mathscr{H}^{n-1}\right)$ for $1<p<\infty$ if $b \in \operatorname{VMO}\left(\mathscr{H}^{n-1}\right)$.

However, to obtain reverse arguments of (5) and (6) above, we still need to know more about the pointwise lower bound of the kernel $K$. To obtain the reverse of (5) we aim at verifying the kernel lower bound established by Hytönen [19]. To obtain the reverse of (6) we aim at verifying the same pointwise lower bound as in [5] in a twisted truncated sector.

Theorem 1.4. The Cauchy-Szegö kernel $K(\cdot, \cdot)$ on $\mathscr{H}^{n-1}$ satisfies the following pointwise lower bound: there exist a large positive constant $r_{0}$ and a positive constant $C$ such that for every $g \in \mathscr{H}^{n-1}$, there exists a "twisted truncated sector" $S_{g} \subset \mathscr{H}^{n-1}$ such that

$$
\inf _{g^{\prime} \in S_{g}} \rho\left(g, g^{\prime}\right)=r_{0}
$$

and that for every $g_{1} \in B(g, 1)$ and $g_{2} \in S_{g}$ we have

$$
\left|K\left(g_{1}, g_{2}\right)\right| \geq \frac{C}{\rho\left(g_{1}, g_{2}\right)^{Q}} .
$$

Moreover, this sector $S_{g}$ is regular in the sense that $\left|S_{g}\right|=\infty$ and that for every $R_{2}>R_{1}>$ $2 r_{0}$

$$
\left|\left(B\left(g, R_{2}\right) \backslash B\left(g, R_{1}\right)\right) \cap S_{g}\right| \approx\left|B\left(g, R_{2}\right) \backslash B\left(g, R_{1}\right)\right|
$$

with the implicit constants independent of $g$ and $R_{1}, R_{2}$.

By using Theorem 1.4, we now establish the boundedness and compactness of the commutator of $\mathcal{C}$ with respect to $\operatorname{BMO}\left(\mathscr{H}^{n-1}\right)$ and $\operatorname{VMO}\left(\mathscr{H}^{n-1}\right)$, following the ideas and approaches in [19] (see also [11]) and in [5, 13], respectively. 
Theorem 1.5. Suppose $1<p<\infty$ and $b \in L_{l o c}^{1}\left(\mathscr{H}^{n-1}\right)$.

(i) $b \in \operatorname{BMO}\left(\mathscr{H}^{n-1}\right)$ if and only if $[b, \mathcal{C}]$ is bounded on $L^{p}\left(\mathscr{H}^{n-1}\right)$.

(ii) $b \in \operatorname{VMO}\left(\mathscr{H}^{n-1}\right)$ if and only if $[b, \mathcal{C}]$ is compact on $L^{p}\left(\mathscr{H}^{n-1}\right)$.

Thus, we will only sketch the main approach of the proof of Theorem 1.5.

In the next section we will provide some necessary preliminaries on quaternionic Heisenberg groups, and in the last section we provide the proofs of our main results.

\section{Preliminaries}

Recall that the space $\mathbb{H}$ of quaternion numbers forms a division algebra with respect to the coordinate addition and the quaternion multiplication

$$
\begin{aligned}
x x^{\prime}= & \left(x_{1}+x_{2} \mathbf{i}+x_{3} \mathbf{j}+x_{4} \mathbf{k}\right)\left(x_{1}^{\prime}+x_{2}^{\prime} \mathbf{i}+x_{3}^{\prime} \mathbf{j}+x_{4}^{\prime} \mathbf{k}\right) \\
= & x_{1} x_{1}^{\prime}-x_{2} x_{2}^{\prime}-x_{3} x_{3}^{\prime}-x_{4} x_{4}^{\prime}+\left(x_{1} x_{2}^{\prime}+x_{2} x_{1}^{\prime}+x_{3} x_{4}^{\prime}-x_{4} x_{3}^{\prime}\right) \mathbf{i} \\
& +\left(x_{1} x_{3}^{\prime}-x_{2} x_{4}^{\prime}+x_{3} x_{1}^{\prime}+x_{4} x_{2}^{\prime}\right) \mathbf{j}+\left(x_{1} x_{4}^{\prime}+x_{2} x_{3}^{\prime}-x_{3} x_{2}^{\prime}+x_{4} x_{1}^{\prime}\right) \mathbf{k},
\end{aligned}
$$

for any $x=x_{1}+x_{2} \mathbf{i}+x_{3} \mathbf{j}+x_{4} \mathbf{k}, x^{\prime}=x_{1}^{\prime}+x_{2}^{\prime} \mathbf{i}+x_{3}^{\prime} \mathbf{j}+x_{4}^{\prime} \mathbf{k} \in \mathbb{H}$. The conjugate $\bar{x}$ is defined by

$$
\bar{x}=x_{1}-x_{2} \mathbf{i}-x_{3} \mathbf{j}-x_{4} \mathbf{k},
$$

and the modulus $|x|$ is defined by

$$
|x|^{2}=x \bar{x}=\sum_{j=1}^{4} x_{j}^{2} .
$$

The conjugation inverses the product of quaternion number in the following sense $\overline{q \sigma}=\bar{\sigma} \bar{q}$ for any $q, \sigma \in \mathbb{H}$. It is clear that

$$
\begin{aligned}
\operatorname{Im}\left(\bar{x} x^{\prime}\right)= & \operatorname{Im}\left\{\left(x_{1}-x_{2} \mathbf{i}-x_{3} \mathbf{j}-x_{4} \mathbf{k}\right)\left(x_{1}^{\prime}+x_{2}^{\prime} \mathbf{i}+x_{3}^{\prime} \mathbf{j}+x_{4}^{\prime} \mathbf{k}\right)\right\} \\
= & \left(x_{1} x_{2}^{\prime}-x_{2} x_{1}^{\prime}-x_{3} x_{4}^{\prime}+x_{4} x_{3}^{\prime}\right) \mathbf{i}+\left(x_{1} x_{3}^{\prime}+x_{2} x_{4}^{\prime}-x_{3} x_{1}^{\prime}-x_{4} x_{2}^{\prime}\right) \mathbf{j} \\
& +\left(x_{1} x_{4}^{\prime}-x_{2} x_{3}^{\prime}+x_{3} x_{2}^{\prime}-x_{4} x_{1}^{\prime}\right) \mathbf{k} \\
= & : \sum_{\alpha=1}^{3} \sum_{k, j=1}^{4} b_{k j}^{\alpha} x_{k} x_{j}^{\prime} \mathbf{i}_{\alpha},
\end{aligned}
$$

where $\mathbf{i}_{1}=\mathbf{i}, \mathbf{i}_{2}=\mathbf{j}, \mathbf{i}_{3}=\mathbf{k}$, and $b_{k j}^{\alpha}$ is the $(k, j)$ th entry of the following matrices $b^{\alpha}$ :

$$
b^{1}:=\left(\begin{array}{cccc}
0 & 1 & 0 & 0 \\
-1 & 0 & 0 & 0 \\
0 & 0 & 0 & -1 \\
0 & 0 & 1 & 0
\end{array}\right), \quad b^{2}:=\left(\begin{array}{cccc}
0 & 0 & 1 & 0 \\
0 & 0 & 0 & 1 \\
-1 & 0 & 0 & 0 \\
0 & -1 & 0 & 0
\end{array}\right), \quad b^{3}:=\left(\begin{array}{cccc}
0 & 0 & 0 & 1 \\
0 & 0 & -1 & 0 \\
0 & 1 & 0 & 0 \\
-1 & 0 & 0 & 0
\end{array}\right) .
$$

The quaternion space $\mathbb{H}^{n}$ is isomorphic to $\mathbb{R}^{4 n}$ as a real vector space and the pure imaginary $\operatorname{Im} \mathbb{H}$ are isomorphic to $\mathbb{R}^{3}$.

The quaternionic Heisenberg group $\mathscr{H}^{n-1}$ is the space $\mathbb{R}^{4 n-1}=\mathbb{R}^{3} \times \mathbb{R}^{4(n-1)}$, which is isomorphic to $\operatorname{Im} \mathbb{H} \times \mathbb{H}^{n-1}$ endowed with the non-commutative product

$$
(t, y) \cdot\left(t^{\prime}, y^{\prime}\right)=\left(t+t^{\prime}+2 \operatorname{Im}\left\langle y, y^{\prime}\right\rangle, y+y^{\prime}\right),
$$

where $t=t_{1} \mathbf{i}+t_{2} \mathbf{j}+t_{3} \mathbf{k}, t^{\prime}=t_{1}^{\prime} \mathbf{i}+t_{2}^{\prime} \mathbf{j}+t_{3}^{\prime} \mathbf{k} \in \operatorname{Im} \mathbb{H}, y, y^{\prime} \in \mathbb{H}^{n-1}$, and $\langle\cdot, \cdot\rangle$ is the inner product defined by

$$
\left\langle y, y^{\prime}\right\rangle=\sum_{l=1}^{n-1} \bar{y}_{l} y_{l}^{\prime}, \quad y=\left(y_{1}, \cdots, y_{n-1}\right), \quad y^{\prime}=\left(y_{1}^{\prime}, \cdots, y_{n-1}^{\prime}\right) \in \mathbb{H}^{n-1} .
$$


It is easy to check that the identity of $\mathscr{H}^{n-1}$ is the origin $\mathbf{0}:=(0,0)$, and the inverse of $(t, y)$ is given by $(-t,-y)$.

The boundary of quaternionic Siegel upper half-space $\partial \mathcal{U}_{n}$ can be identified with the quaternionic Heisenberg group $\mathscr{H}^{n-1}$ via the projection

$$
\begin{aligned}
\pi: \partial \mathcal{U}_{n} & \longrightarrow \operatorname{Im} \mathbb{H} \times \mathbb{H}^{n-1}, \\
\left(\left|q^{\prime}\right|^{2}+x_{2} \mathbf{i}+x_{3} \mathbf{j}+x_{4} \mathbf{k}, q^{\prime}\right) & \longmapsto\left(x_{2} \mathbf{i}+x_{3} \mathbf{j}+x_{4} \mathbf{k}, q^{\prime}\right) .
\end{aligned}
$$

Let $d \beta$ be the Lebesgue measure on $\partial \mathcal{U}_{n}$ obtained by pulling back the Haar measure on the group $\mathscr{H}^{n-1}$ by the projection $\pi$.

The Hardy space $\mathcal{H}^{2}\left(\mathcal{U}_{n}\right)$ consists of all regular functions $F$ on $\mathcal{U}_{n}$, for which

$$
\|F\|_{\mathcal{H}^{2}\left(\mathcal{U}_{n}\right)}:=\left(\sup _{\varepsilon>0} \int_{\partial \mathcal{U}_{n}}\left|F_{\varepsilon}(q)\right|^{2} d \beta(q)\right)^{\frac{1}{2}}<\infty .
$$

According to [4, Theorem 4.1], a function $F \in \mathcal{H}^{2}\left(\mathcal{U}_{n}\right)$ has boundary value $F^{b}$ that belongs to $L^{2}\left(\mathcal{U}_{n}\right)$.

By (2.1), the multiplication of the quaternionic Heisenberg group in terms of real variables can be written as (cf. [29])

$$
(t, y) \cdot\left(t^{\prime}, y^{\prime}\right)=\left(t_{\alpha}+t_{\alpha}^{\prime}+2 \sum_{l=0}^{n-1} \sum_{j, k=1}^{4} b_{k j}^{\alpha} y_{4 l+k} y_{4 l+j}^{\prime}, y+y^{\prime}\right),
$$

where $t=\left(t_{1}, t_{2}, t_{3}\right), t^{\prime}=\left(t_{1}^{\prime}, t_{2}^{\prime}, t_{3}^{\prime}\right) \in \mathbb{R}^{3}, \alpha=1,2,3, y=\left(y_{1}, y_{2}, \cdots, y_{4 n-4}\right), y^{\prime}=$ $\left(y_{1}^{\prime}, y_{2}^{\prime}, \cdots, y_{4 n-4}^{\prime}\right) \in \mathbb{R}^{4 n-4}$.

The following vector fields are left invariant on the quaternionic Heisenberg group by the multiplication laws of the quaternionic Heisenberg group in (2.4):

$$
Y_{4 l+j}=\frac{\partial}{\partial y_{4 l+j}}+2 \sum_{\alpha=1}^{3} \sum_{k=1}^{4} b_{k j}^{\alpha} y_{4 l+k} \frac{\partial}{\partial t_{\alpha}},
$$

and

$$
\left[Y_{4 l^{\prime}+k}, Y_{4 l+j}\right]=2 \delta_{l l^{\prime}} \sum_{\alpha=1}^{3} b_{k j}^{\alpha} \frac{\partial}{\partial t_{\alpha}},
$$

for $l, l^{\prime}=0, \cdots, n-2, j, k=1, \cdots 4$. Then the horizontal tangent space at $g \in \mathscr{H}^{n-1}$, denoted by $H_{g}$, is spanned by the left invariant vectors $Y_{1}(g), \cdots, Y_{4 n-4}(g)$. For each $g \in$ $\mathscr{H}^{n-1}$, we fix a quadratic form $\langle\cdot, \cdot\rangle_{H}$ on $H_{g}$ with respect to which the vectors $Y_{1}(g), \cdots, Y_{4 n-4}(g)$ are orthonormal.

For any $p=(t, y) \in \mathscr{H}^{n-1}$, we can associate the automorphism $\tau_{p}$ of $\mathcal{U}_{n}$ :

$$
\tau_{p}:\left(q_{1}, q^{\prime}\right) \longmapsto\left(q_{1}+|y|^{2}+t+2\left\langle y, q^{\prime}\right\rangle, q^{\prime}+y\right) .
$$

It is obviously extended to the boundary $\partial \mathcal{U}_{n}$. It is easy to see that the action on $\partial \mathcal{U}_{n}$ is transitive. In particular, we have

$$
\tau_{p}:(0,0) \longmapsto\left(|y|^{2}+t, y\right) .
$$

And we can write each $q \in \partial \mathcal{U}_{n}$ as $q=\tau_{g}(0)$ for a unique $g \in \mathscr{H}^{n-1}$. In this correspondence we have that $d \beta(q)=d g$, the invariant measure on $\mathscr{H}^{n-1}$. Similarly, we write $p \in \partial \mathcal{U}_{n}$ in the form $p=\tau_{h}(0)$ for some unique $h \in \mathscr{H}^{n-1}$. Then from (2.6) we can see that

$$
S(q+\varepsilon \mathbf{e}, p)=S\left(\tau_{h^{-1}}(q+\varepsilon \mathbf{e}), \tau_{h^{-1}}(p)\right)=S\left(\tau_{h^{-1}}(q)+\varepsilon \mathbf{e}, 0\right)=S\left(\tau_{h^{-1} \cdot g}(0)+\varepsilon \mathbf{e}, 0\right) .
$$


Take $K_{\varepsilon}(g)=S\left(\tau_{g}(0)+\varepsilon \mathbf{e}, 0\right)$, and denote by $f(h):=f\left(\tau_{h}(0)\right)=f(p)$ and $(\mathcal{C} f)(g):=$ $(\mathcal{C} f)\left(\tau_{g}(0)\right)=(\mathcal{C} f)(q)$ by abuse of notations. Then

$$
(\mathcal{C} f)(g)=\lim _{\varepsilon \rightarrow 0} \int_{\mathscr{H}^{n-1}} K_{\varepsilon}\left(h^{-1} \cdot g\right) f(h) d h, \quad f \in L^{2}\left(\mathscr{H}^{n-1}\right),
$$

where the limit is taken in $L^{2}\left(\mathscr{H}^{n-1}\right)$.

Recall that the convolution on $\mathscr{H}^{n-1}$ is defined as

$$
(f * \tilde{f})(g)=\int_{\mathscr{H}^{n-1}} f(h) \tilde{f}\left(h^{-1} \cdot g\right) d h .
$$

Therefore, (2.7) can be formally rewritten as

$$
(\mathcal{C} f)(g)=(f * K)(g),
$$

where $K$ is the distribution given by $\lim _{\varepsilon \rightarrow 0} K_{\varepsilon}$. Thus, if $g=(t, y) \in \mathscr{H}^{n-1}$ with $t=$ $t_{1} \mathbf{i}+t_{2} \mathbf{j}+t_{3} \mathbf{k}$, then

$$
K(g)=\lim _{\varepsilon \rightarrow 0} K_{\varepsilon}(g)=s\left(|y|^{2}+t\right) .
$$

For any $g=(t, y) \in \mathscr{H}^{n-1}$, the homogeneous norm of $g$ is defined by

$$
\|g\|=\left(|y|^{4}+\sum_{j=1}^{3}\left|t_{j}\right|^{2}\right)^{\frac{1}{4}} .
$$

Obviously, $\left\|g^{-1}\right\|=\|-g\|=\|g\|$ and $\left\|\delta_{r}(g)\right\|=r\|g\|$, where $\delta_{r}, r>0$, is the dilation on $\mathscr{H}^{n-1}$, which is defined as

$$
\delta_{r}(t, y)=\left(r^{2} t, r y\right) .
$$

On $\mathscr{H}^{n-1}$, we define the quasi-distance

$$
\rho(h, g)=\left\|g^{-1} \cdot h\right\| .
$$

It is clear that $\rho$ is symmetric and satisfies the generalized triangle inequality

$$
\rho(h, g) \leq C_{\rho}(\rho(h, w)+\rho(w, g)),
$$

for any $h, g, w \in \mathscr{H}^{n-1}$ and some $C_{\rho}>0$. Using $\rho$, we define the balls $B(g, r)$ in $\mathscr{H}^{n-1}$ by $B(g, r)=\{h: \rho(h, g)<r\}$. Then

$$
|B(g, r)| \approx r^{Q} .
$$

We now recall the Hardy, BMO and VMO spaces. Note that $\mathscr{H}^{n-1}$ falls into the scope of homogeneous group, and hence we have the natural BMO space (also the Hardy space) in this setting due to Folland and Stein [10]. To be self-enclosed, we recall the definition of the BMO space.

$$
\operatorname{BMO}\left(\mathscr{H}^{n-1}\right)=\left\{b \in L_{l o c}^{1}\left(\mathscr{H}^{n-1}\right):\|b\|_{\mathrm{BMO}\left(\mathscr{H}^{n-1}\right)}<\infty\right\},
$$

where

$$
\|b\|_{\mathrm{BMO}\left(\mathscr{C}^{n-1}\right)}=\sup _{B} \frac{1}{|B|} \int_{B}\left|b(g)-b_{B}\right| d g,
$$

where the supremum is taken over all balls $B \subset \mathscr{H}^{n-1}$, and $b_{B}$ is the average of $b$ over the ball $B$. Similarly we also have the VMO space on $\mathscr{H}^{n-1}$, which is closure of the $C_{c}^{\infty}\left(\mathscr{H}^{n-1}\right)$ under the norm of $\|\cdot\|_{\mathrm{BMO}\left(\mathscr{H}^{n-1}\right)}$, see [5] for more details of the definition and properties of this VMO space in the more general setting, the stratified Lie group. 


\section{Proof of MAin Results}

Proof of Theorem 1.1. Note that for $\sigma=x_{1}+x_{2} \mathbf{i}+x_{3} \mathbf{j}+x_{4} \mathbf{k}$, we have $|\sigma|^{2}=x_{1}^{2}+x_{2}^{2}+x_{3}^{2}+x_{4}^{2}$, and $\bar{\sigma}=x_{1}-x_{2} \mathbf{i}-x_{3} \mathbf{j}-x_{4} \mathbf{k}$. By Theorem A,

$$
\begin{aligned}
s(\sigma) & =c_{n-1} \frac{\partial^{2(n-1)}}{\partial x_{1}^{2(n-1)}} \frac{\bar{\sigma}}{|\sigma|^{4}} \\
& =c_{n-1} \frac{\partial^{2(n-1)}}{\partial x_{1}^{2(n-1)}}\left(\frac{1}{|\sigma|^{4}}\right) \bar{\sigma}+(2 n-2) c_{n-1} \frac{\partial^{2 n-3}}{\partial x_{1}^{2 n-3}}\left(\frac{1}{|\sigma|^{4}}\right) .
\end{aligned}
$$

Now it suffices to figure out the representation of

$$
\frac{\partial^{l}}{\partial x_{1}^{l}}\left(\frac{1}{|\sigma|^{4}}\right)
$$

where $l$ is any fixed natural number.

To see this, let $z=x_{1}+|\operatorname{Im} \sigma| \mathbf{i}$, and then $\bar{z}=x_{1}-|\operatorname{Im} \sigma| \mathbf{i}$. Hence, we further have

$$
|\sigma|^{2}=z \bar{z} .
$$

As a consequence, we have

$$
\frac{\partial^{l}}{\partial x_{1}^{l}}\left(\frac{1}{|\sigma|^{4}}\right)=\frac{\partial^{l}}{\partial x_{1}^{l}}\left(\frac{1}{z^{2} \bar{z}^{2}}\right)
$$

By using the binomial expansion, we obtain that

$$
\frac{\partial^{l}}{\partial x_{1}^{l}}\left(\frac{1}{|\sigma|^{4}}\right)=\sum_{k=0}^{l} C_{l}^{k} \frac{\partial^{l-k}}{\partial x_{1}^{l-k}}\left(\frac{1}{z^{2}}\right) \frac{\partial^{k}}{\partial x_{1}^{k}}\left(\frac{1}{\bar{z}^{2}}\right),
$$

where

$$
C_{l}^{k}=\frac{l !}{k !(l-k) !} .
$$

Next, by collating the coefficients, we further have

$$
\frac{\partial^{l}}{\partial x_{1}^{l}}\left(\frac{1}{|\sigma|^{4}}\right)=\sum_{k=0}^{l}(-1)^{l} l !(l-k+1)(k+1)\left(\frac{1}{z^{l-k+2}}\right)\left(\frac{1}{\bar{z}^{k+2}}\right) .
$$

Then the key step is to obtain an explicit formula for the above summation. To see this, we rewrite the right-hand side of (3.2) as follows.

$$
\text { RHS of }(\underline{3.2})=\frac{(-1)^{l} l !}{z^{l}|z|^{4}} \sum_{k=0}^{l}(l-k+1)(k+1)\left(\frac{z}{\bar{z}}\right)^{k}=: \frac{(-1)^{l} l !}{z^{l}|z|^{4}} \mathbf{A}\left(\frac{z}{\bar{z}}\right) \text {. }
$$

Now let $w=\frac{z}{\bar{z}}$, then

$$
\mathbf{A}(w)=\sum_{k=0}^{l}(l-k+1)(k+1) w^{k} .
$$

Based on the definition of $\mathbf{A}(w)$, we now consider the following function

$$
\mathbf{G}(w):=\sum_{k=0}^{l}(l-k+1) w^{k+1} .
$$

It is obvious that

$$
\frac{d}{d w} \mathbf{G}(w)=\mathbf{A}(w) .
$$


To continue, we now set $\zeta=w^{-1}$. Then by changing of variable we have

$$
\mathbf{G}\left(\frac{1}{\zeta}\right)=\sum_{k=0}^{l}(l-k+1) \zeta^{-k-1} .
$$

Next, we set

$$
\mathbf{H}(\zeta):=\zeta^{l+1} \mathbf{G}\left(\frac{1}{\zeta}\right)=\sum_{k=0}^{l}(l-k+1) \zeta^{l-k} .
$$

Again, based on the definition of $\mathbf{H}$, we now consider the following function

$$
\mathbf{F}(\zeta):=\sum_{k=0}^{l} \zeta^{l-k+1}
$$

and it is obvious that

$$
\frac{d}{d \zeta} \mathbf{F}(\zeta)=\mathbf{H}(\zeta)
$$

Now by taking the summation we have

$$
\mathbf{F}(\zeta)=\zeta^{l+1} \sum_{k=0}^{l} \zeta^{-k}=\frac{\zeta\left(\zeta^{l+1}-1\right)}{\zeta-1},
$$

and hence

$$
\mathbf{H}(\zeta)=\frac{d}{d \zeta}\left(\frac{\zeta\left(\zeta^{l+1}-1\right)}{\zeta-1}\right)=\frac{(l+1) \zeta^{l+2}-(l+2) \zeta^{l+1}+1}{(\zeta-1)^{2}} .
$$

As a consequence, we get that

$$
\mathbf{G}(w)=\frac{(l+1) w}{(1-w)^{2}}-\frac{(l+2) w^{2}}{(1-w)^{2}}+\frac{w^{l+3}}{(1-w)^{2}} .
$$

We now have

$$
\begin{aligned}
\mathbf{A}(w) & =\frac{d}{d w}\left(\frac{(l+1) w}{(1-w)^{2}}-\frac{(l+2) w^{2}}{(1-w)^{2}}+\frac{w^{l+3}}{(1-w)^{2}}\right) \\
& =\frac{(l+1)-(l+3) w+(l+3) w^{l+2}-(l+1) w^{l+3}}{(1-w)^{3}} .
\end{aligned}
$$

By changing $w$ back to $\frac{z}{\bar{z}}$, we have

$$
\mathbf{A}\left(\frac{z}{\bar{z}}\right)=\frac{(l+1) \frac{z^{l+3}}{\bar{z}^{l}}-(l+3) \frac{z^{l+2}}{\bar{z}^{l-1}}+(l+3) z \bar{z}^{2}-(l+1) \bar{z}^{3}}{(z-\bar{z})^{3}}
$$

Hence,

$$
\begin{aligned}
\text { RHS of }(\underline{3.2)}) & =\frac{(-1)^{l} l !}{|z|^{4}} \frac{(l+1) \frac{z^{3}}{\bar{z}^{l}}-(l+3) \frac{z^{2}}{\bar{z}^{l-1}}+(l+3) \frac{\bar{z}^{2}}{z^{l-1}}-(l+1) \frac{\bar{z}^{3}}{z^{l}}}{(z-\bar{z})^{3}} \\
& =\frac{(-1)^{l} l !}{|z|^{4}(z-\bar{z})^{3}}\left(-2(l+1) \operatorname{Im}\left(\frac{\bar{z}^{3}}{z^{l}}\right) \mathbf{i}+2(l+3) \operatorname{Im}\left(\frac{\bar{z}^{2}}{z^{l-1}}\right) \mathbf{i}\right) \\
& =\frac{4(-1)^{l} l !}{|z|^{4}(z-\bar{z})^{3}} \operatorname{Im}\left[\frac{\bar{z}^{2}}{z^{l}}\left(z+(l+1) \frac{z-\bar{z}}{2}\right)\right] \mathbf{i} .
\end{aligned}
$$

Based on the representation in (3.2) above, from (3.1), we obtain that

$$
s(\sigma)=c_{n-1} \frac{4(2 n-2) !}{|z|^{4}(z-\bar{z})^{3}} \operatorname{Im}\left[\frac{\bar{z}^{2}}{z^{2 n-2}}\left(z+(2 n-1) \frac{z-\bar{z}}{2}\right)\right] \mathbf{i} \bar{\sigma}
$$




$$
\begin{aligned}
& -(2 n-2) c_{n-1} \frac{4(2 n-3) !}{|z|^{4}(z-\bar{z})^{3}} \operatorname{Im}\left[\frac{\bar{z}^{2}}{z^{2 n-3}}\left(z+(2 n-2) \frac{z-\bar{z}}{2}\right)\right] \mathbf{i} \\
= & c_{n-1} \frac{4(2 n-2) !}{|z|^{4}(z-\bar{z})^{3}}\left\{\operatorname{Im}\left[\frac{\bar{z}^{2}}{z^{2 n-2}}\left(z+(2 n-1) \frac{z-\bar{z}}{2}\right)\right] \mathbf{i} \bar{\sigma}\right. \\
& \left.-\operatorname{Im}\left[\frac{\bar{z}^{2}}{z^{2 n-3}}\left(z+(2 n-2) \frac{z-\bar{z}}{2}\right)\right] \mathbf{i}\right\} .
\end{aligned}
$$

The proof of Theorem 1.1 is complete.

Based on the result of Theorem 1.1 we see that the kernel $K$ of the Cauchy-Szegö projection operator $\mathcal{C}$ on $\mathscr{H}^{n-1}$ is homogeneous. To be more specific, we have the following.

Corollary 3.1. The kernel $K$ of the Cauchy-Szegö projection operator $\mathcal{C}$ on $\mathscr{H}^{n-1}$ satisfies

$$
K\left(\delta_{r}(g)\right)=r^{-Q} K(g)
$$

for every $g \in \mathscr{H}^{n-1}$ and $r>0$.

Before proving Theorem 1.2, we will need a mean value theorem on stratified groups. Note that there is a version established in [10, (1.41)]. However it requires the function $f \in$ $C^{1}\left(\mathscr{H}^{n-1}\right)$, while the kernel $K$ of the Cauchy-Szegö projection operator $\mathcal{C}$ has singularity at the origin.

We now provide the following mean value theorem on stratified groups with respect to the kernel $K$. To begin with we recall that an absolutely continuous curve $\gamma:[0,1] \rightarrow \mathscr{H}^{n-1}$ is horizontal if its tangent vectors $\dot{\gamma}(t), t \in[0,1]$, lie in the horizontal tangent space $H_{\gamma(t)}$. By [6], any given two points $p, q \in \mathscr{H}^{n-1}$ can be connected by a horizontal curve.

The Carnot-Carathéodory metric on $\mathscr{H}^{n-1}$ as follows. For $g, h \in \mathscr{H}^{n-1}$,

$$
d_{c c}(g, h):=\inf _{\gamma} \int_{0}^{1}\langle\dot{\gamma}(t), \dot{\gamma}(t)\rangle_{H}^{\frac{1}{2}} d t,
$$

where $\gamma:[0,1] \rightarrow \mathscr{H}^{n-1}$ is a horizontal Lipschitz curve with $\gamma(0)=g, \gamma(1)=h$. It is known that the Carnot-Carathéodory metric $d_{c c}$ is left-invariant, and it is equivalent to the homogeneous metric $\rho$ in the sense that: there exist $C_{d}, \tilde{C}_{d}>0$ such that for any $g, h \in \mathscr{H}^{n-1}($ see $[2])$,

$$
C_{d} \rho(g, h) \leq d_{c c}(g, h) \leq \tilde{C}_{d} \rho(g, h) .
$$

Lemma 3.2. There exist $C>0$ and $c_{0} \in(0,1)$ such that for $g, g_{0} \in \mathscr{H}^{n-1} \backslash\{\mathbf{0}\}$ with $d_{c c}\left(g, g_{0}\right)<c_{0} d_{c c}\left(g_{0}, \mathbf{0}\right)$, we have

$$
\left|K(g)-K\left(g_{0}\right)\right| \leq C d_{c c}\left(g, g_{0}\right) \times \max _{\substack{1 \leq j \leq 4 n-4 \\ u: d_{c c}(u, 0) \leq d_{c c}\left(g, g_{0}\right)}}\left|Y_{j} K\left(g_{0} \cdot u\right)\right| .
$$

Proof. Suppose that $g, g_{0} \in \mathscr{H}^{n-1} \backslash\{\mathbf{0}\}$ with $d_{c c}\left(g, g_{0}\right)<c_{0} d_{c c}\left(g_{0}, \mathbf{0}\right)$, and let $\gamma$ be the curve in the definition of (3.4) with $\gamma(0)=g$ and $\gamma(1)=g_{0}$ such that

$$
d_{c c}\left(g, g_{0}\right) \leq \int_{0}^{1}\langle\dot{\gamma}(t), \dot{\gamma}(t)\rangle_{H}^{\frac{1}{2}} d t \leq \frac{101}{100} d_{c c}\left(g, g_{0}\right) .
$$

Then for any $t \in[0,1]$, we have

$$
\begin{aligned}
d_{c c}(\gamma(t), \mathbf{0}) & \geq d_{c c}\left(g_{0}, \mathbf{0}\right)-d_{c c}\left(\gamma(t), g_{0}\right) \geq d_{c c}\left(g_{0}, \mathbf{0}\right)-c_{0} d_{c c}\left(g_{0}, \mathbf{0}\right) \\
& =\left(1-c_{0}\right) d_{c c}\left(g_{0}, \mathbf{0}\right) .
\end{aligned}
$$

So $K(\gamma(t))$ is not singular for every $t \in[0,1]$. 
To continue, we now write $\gamma(t)=\left(\gamma_{1}(t), \ldots, \gamma_{4 n-1}(t)\right) \in \mathscr{H}^{n-1}$. Then

$$
\dot{\gamma}(t)=\sum_{j=1}^{4 n-4} \gamma_{j}^{\prime}(t) \frac{\partial}{\partial y_{j}}+\sum_{\alpha=1}^{3} \gamma_{4 n-4+\alpha}^{\prime}(t) \frac{\partial}{\partial t_{\alpha}}=\sum_{j=1}^{4 n-4} a_{j}(t) Y_{j}(\gamma(t)),
$$

where $a_{j}(t):=\gamma_{j}^{\prime}(t)$ for $j=1,2, \ldots, 4 n-4$,

$$
\gamma_{4 n-4+\alpha}^{\prime}(t)=2 \sum_{l=1}^{n-2} \sum_{j=1}^{4} \sum_{k=1}^{4} a_{4 l+j} b_{k j}^{\alpha} \gamma_{4 l+k}(t),
$$

and $\langle\dot{\gamma}(t), \dot{\gamma}(t)\rangle_{H}=\sum_{j=1}^{4 n-4}\left|a_{j}(t)\right|^{2}$.

As a consequence, we have

$$
\begin{aligned}
K(g)-K\left(g_{0}\right) & =\int_{0}^{1} \frac{d}{d t}(K(\gamma(t))) d t=\int_{0}^{1}\left(\sum_{j=1}^{4 n-4} \frac{\partial K}{\partial y_{j}} \gamma_{j}^{\prime}(t)+\sum_{\alpha=1}^{3} \frac{\partial K}{\partial t_{\alpha}} \gamma_{4 n-4+\alpha}^{\prime}(t)\right) d t \\
& =\int_{0}^{1} \sum_{j=1}^{4 n-4} a_{j}(t) Y_{j} K(\gamma(t)) d t .
\end{aligned}
$$

Hence,

$$
\begin{aligned}
& \left|K(g)-K\left(g_{0}\right)\right| \leq \max _{\substack{1 \leq j \leq 4 n-4 \\
0 \leq t \leq 1}}\left|Y_{j} K(\gamma(t))\right| \int_{0}^{1} \sum_{j=1}^{4 n-4}\left|a_{j}(t)\right| d t \\
& \leq C \max _{\substack{1 \leq j \leq 4 n-4 \\
0 \leq t \leq 1}}\left|Y_{j} K(\gamma(t))\right| \int_{0}^{1}\left(\sum_{j=1}^{4 n-4}\left|a_{j}(t)\right|^{2}\right)^{\frac{1}{2}} d t \\
& =C \max _{\substack{1 \leq j \leq 4 n-4 \\
0 \leq t \leq 1}}\left|Y_{j} K(\gamma(t))\right| \int_{0}^{1}\langle\dot{\gamma}(t), \dot{\gamma}(t)\rangle^{\frac{1}{2}} d t \\
& \leq C \max _{\substack{1 \leq j \leq 4 n-4 \\
0 \leq t \leq 1}}\left|Y_{j} K(\gamma(t))\right| d_{c c}\left(g, g_{0}\right)
\end{aligned}
$$

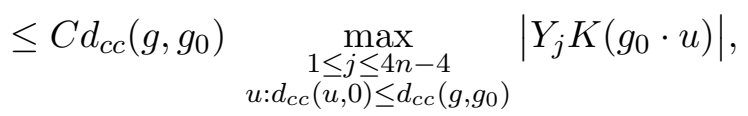

where $C$ is an absolute constant depending only on $n$.

The proof of Lemma 3.2 is complete.

Proof of Theorem 1.2. We begin with proving the size estimate in (i).

By (2.8), for any $g=(t, y) \in \mathscr{H}^{n-1}$, where $t=t_{1} \mathbf{i}+t_{2} \mathbf{j}+t_{3} \mathbf{k}, y \in \mathbb{H}^{n-1}$, we have

$$
K(g)=s\left(|y|^{2}+t\right) .
$$

From (3.1) and (3.2), we can see that for $\sigma=x_{1}+x_{2} \mathbf{i}+x_{3} \mathbf{j}+x_{4} \mathbf{k}$,

$$
\begin{aligned}
s(\sigma)= & c_{n-1} \sum_{k=0}^{2 n-2}(2 n-2) !(2 n-k-1)(k+1) \frac{\bar{\sigma}}{z^{2 n-k} \bar{z}^{k+2}} \\
& -c_{n-1} \sum_{k=0}^{2 n-3}(2 n-2) !(2 n-k-2)(k+1) \frac{1}{z^{2 n-k-1} \bar{z}^{k+2}},
\end{aligned}
$$

where $z=x_{1}+|\operatorname{Im} \sigma|$ i. Therefore,

$$
K(g)=s\left(|y|^{2}+t\right)
$$




$$
\begin{aligned}
= & c_{n-1} \sum_{k=0}^{2 n-2}(2 n-2) !(2 n-k-1)(k+1) \frac{|y|^{2}-t}{\left(|y|^{2}+|t| \mathbf{i}\right)^{2 n-k}\left(|y|^{2}-|t| \mathbf{i}\right)^{k+2}} \\
& -c_{n-1} \sum_{k=0}^{2 n-3}(2 n-2) !(2 n-k-2)(k+1) \frac{1}{\left(|y|^{2}+|t| \mathbf{i}\right)^{2 n-k-1}\left(|y|^{2}-|t| \mathbf{i}\right)^{k+2}} .
\end{aligned}
$$

Since

$$
\begin{aligned}
\left|\frac{|y|^{2}-t}{\left(|y|^{2}+|t| \mathbf{i}\right)^{2 n-k}\left(|y|^{2}-|t| \mathbf{i}\right)^{k+2} \mid}\right| & =\frac{\left(|y|^{4}+|t|^{2}\right)^{\frac{1}{2}}}{\left(|y|^{4}+|t|^{2}\right)^{\frac{2 n-k}{2}}\left(|y|^{4}+|t|^{2}\right)^{\frac{k+2}{2}}} \\
& =\frac{1}{\left(|y|^{4}+|t|^{2}\right)^{\frac{2 n+1}{2}}}=\frac{1}{\|g\|^{Q}},
\end{aligned}
$$

and

$$
\left|\frac{1}{\left(|y|^{2}+|t| \mathbf{i}\right)^{2 n-k-1}\left(|y|^{2}-|t| \mathbf{i}\right)^{k+2}}\right|=\frac{1}{\left(|y|^{4}+|t|^{2}\right)^{\frac{2 n+1}{2}}}=\frac{1}{\|g\|^{Q}} .
$$

We have

$$
|K(g)| \lesssim \frac{1}{\|g\|^{Q}},
$$

where the implicit constant depends only on $n$, which shows that (i) holds.

We now prove the regularity estimate as in (ii).

To begin with, we recall that the vector field

$$
Y_{4 l+j}=\frac{\partial}{\partial y_{4 l+j}}+2 \sum_{\alpha=1}^{3} \sum_{k=1}^{4} b_{k j}^{\alpha} y_{4 l+k} \frac{\partial}{\partial t_{\alpha}}, \quad l=0, \cdots, n-2, \quad j=1, \cdots 4,
$$

where for $\alpha=1,2,3, b_{k j}^{\alpha}$ is the element (from the $k$ th row and the $j$ th column) in the matrix $b^{\alpha}$ below:

$$
b^{1}:=\left(\begin{array}{cccc}
0 & 1 & 0 & 0 \\
-1 & 0 & 0 & 0 \\
0 & 0 & 0 & -1 \\
0 & 0 & 1 & 0
\end{array}\right), \quad b^{2}:=\left(\begin{array}{cccc}
0 & 0 & 1 & 0 \\
0 & 0 & 0 & 1 \\
-1 & 0 & 0 & 0 \\
0 & -1 & 0 & 0
\end{array}\right), \quad b^{3}:=\left(\begin{array}{cccc}
0 & 0 & 0 & 1 \\
0 & 0 & -1 & 0 \\
0 & 1 & 0 & 0 \\
-1 & 0 & 0 & 0
\end{array}\right) .
$$

For any $g, h, g_{0}$ with $\rho\left(g_{0}, h\right) \geq c \rho\left(g, g_{0}\right)$, we set $x=h^{-1} \cdot g_{0}$, then by Lemma 3.2 and (3.5), we have

$$
\begin{aligned}
\left|K(g, h)-K\left(g_{0}, h\right)\right| & =\left|K\left(h^{-1} \cdot g\right)-K\left(h^{-1} \cdot g_{0}\right)\right| \\
& =\left|K\left(x \cdot\left(g_{0}^{-1} \cdot g\right)\right)-K(x)\right| \\
& \leq C d_{c c}\left(g, g_{0}\right) \max _{\substack{1 \leq j \leq 4 n-4 \\
u: d_{c c}(u, 0) \leq d_{c c}\left(g, g_{0}\right)}}\left|Y_{j} K(x \cdot u)\right| \\
& \leq C \rho\left(g, g_{0}\right) \max _{\substack{1 \leq j \leq 4 n-4 \\
u: d_{c c}(u, 0) \leq d_{c c}\left(g, g_{0}\right)}}\left|Y_{j} K(x \cdot u)\right| .
\end{aligned}
$$

Recall that for any $g=(t, y)=\left(t_{1} \mathbf{i}+t_{2} \mathbf{j}+t_{3} \mathbf{k}, y\right)$,

$$
K(g)=s\left(|y|^{2}+t\right)=s\left(|y|^{2}, t_{1}, t_{2}, t_{3}\right) .
$$

Then for any $l=0, \cdots, n-2, j=1, \cdots 4$.

$$
Y_{4 l+j} K(g)=\left(\frac{\partial}{\partial y_{4 l+j}}+2 \sum_{\alpha=1}^{3} \sum_{k=1}^{4} b_{k j}^{\alpha} y_{4 l+k} \frac{\partial}{\partial t_{\alpha}}\right) s\left(|y|^{2}, t_{1}, t_{2}, t_{3}\right)
$$




$$
\begin{aligned}
& =\left(\frac{\partial}{\partial y_{4 l+j}}+2 \sum_{\alpha=1}^{3} \sum_{k=1}^{4} b_{k j}^{\alpha} y_{4 l+k} \frac{\partial}{\partial t_{\alpha}}\right) s\left(|y|^{2}, t_{1}, t_{2}, t_{3}\right) \\
& =2 y_{4 l+j} \frac{\partial s}{\partial x_{1}}\left(|y|^{2}, t_{1}, t_{2}, t_{3}\right)+2 \sum_{\alpha=1}^{3} \sum_{k=1}^{4} b_{k j}^{\alpha} y_{4 l+k} \frac{\partial s}{\partial x_{\alpha+1}}\left(|y|^{2}, t_{1}, t_{2}, t_{3}\right)
\end{aligned}
$$

From (3.1) and (3.2), we can see that

$$
\begin{aligned}
\frac{\partial s}{\partial x_{1}}= & c_{n-1} \frac{\partial^{2 n-1}}{\partial x_{1}^{2 n-1}}\left(\frac{1}{|\sigma|^{4}}\right) \bar{\sigma}+c_{n-1} \frac{\partial^{2 n-2}}{\partial x_{1}^{2 n-2}}\left(\frac{1}{|\sigma|^{4}}\right) \frac{\partial \bar{\sigma}}{\partial x_{1}}+(2 n-2) c_{n-1} \frac{\partial^{2 n-2}}{\partial x_{1}^{2 n-2}}\left(\frac{1}{|\sigma|^{4}}\right) \\
= & c_{n-1} \frac{\partial^{2 n-1}}{\partial x_{1}^{2 n-1}}\left(\frac{1}{|\sigma|^{4}}\right) \bar{\sigma}+(2 n-1) c_{n-1} \frac{\partial^{2 n-2}}{\partial x_{1}^{2 n-2}}\left(\frac{1}{|\sigma|^{4}}\right) \\
= & -c_{n-1} \sum_{k=0}^{2 n-1}(2 n-1) !(2 n-k)(k+1) \frac{1}{z^{2 n-k+1} \bar{z}^{k+2}} \bar{\sigma} \\
& +c_{n-1} \sum_{k=0}^{2 n-2}(2 n-1) !(2 n-k-1)(k+1) \frac{1}{z^{2 n-k} \bar{z}^{k+2}},
\end{aligned}
$$

where we used the fact that $\frac{\partial \bar{\sigma}}{\partial x_{1}}=1$.

Next, by (3.6), we have

$$
\begin{aligned}
\frac{\partial s}{\partial x_{\alpha+1}}= & c_{n-1} \sum_{k=0}^{2 n-2}(2 n-2) !(2 n-k-1)(k+1) \frac{\partial}{\partial x_{\alpha+1}}\left[\frac{\bar{\sigma}}{z^{2 n-k} \bar{z}^{k+2}}\right] \\
& -c_{n-1} \sum_{k=0}^{2 n-3}(2 n-2) !(2 n-k-2)(k+1) \frac{\partial}{\partial x_{\alpha+1}}\left[\frac{1}{z^{2 n-k-1} \bar{z}^{k+2}}\right]
\end{aligned}
$$

where

$$
\begin{aligned}
& \frac{\partial}{\partial x_{\alpha+1}}\left[\frac{\bar{\sigma}}{z^{2 n-k} \bar{z}^{k+2}}\right] \\
& =\frac{1}{z^{2 n-k} \bar{z}^{k+2}} \frac{\partial \bar{\sigma}}{\partial x_{\alpha+1}}-\frac{(2 n-k) \bar{\sigma}}{z^{2 n-k+1} \bar{z}^{k+2}} \frac{\partial z}{\partial x_{\alpha+1}}-\frac{(k+2) \bar{\sigma}}{z^{2 n-k} \bar{z}^{k+3}} \frac{\partial \bar{z}}{\partial x_{\alpha+1}} \\
& =-\frac{1}{z^{2 n-k} \bar{z}^{k+2}} \mathbf{i}_{\alpha}-\frac{(2 n-k) \bar{\sigma}}{z^{2 n-k+1} \bar{z}^{k+2}} \frac{x_{\alpha+1}}{|\operatorname{Im} \sigma|} \mathbf{i}_{\alpha}+\frac{(k+2) \bar{\sigma}}{z^{2 n-k} \bar{z}^{k+3}} \frac{x_{\alpha+1}}{|\operatorname{Im} \sigma|} \mathbf{i}_{\alpha}
\end{aligned}
$$

and

$$
\begin{aligned}
\frac{\partial}{\partial x_{\alpha+1}}\left[\frac{1}{z^{2 n-k-1} \bar{z}^{k+2}}\right] & =-\frac{(2 n-k-1)}{z^{2 n-k} \bar{z}^{k+2}} \frac{\partial z}{\partial x_{\alpha+1}}-\frac{k+2}{z^{2 n-k-1} \bar{z}^{k+3}} \frac{\partial \bar{z}}{\partial x_{\alpha+1}} \\
& =-\frac{(2 n-k-1)}{z^{2 n-k} \bar{z}^{k+2}} \frac{x_{\alpha+1}}{|\operatorname{Im} \sigma|} \mathbf{i}_{\alpha}+\frac{k+2}{z^{2 n-k-1} \bar{z}^{k+3}} \frac{x_{\alpha+1}}{|\operatorname{Im} \sigma|} \mathbf{i}_{\alpha} .
\end{aligned}
$$

Therefore,

$$
\begin{aligned}
& Y_{4 l+j} K(g) \\
& =2 y_{4 l+j} \frac{\partial s}{\partial x_{1}}\left(|y|^{2}, t_{1}, t_{2}, t_{3}\right)+2 \sum_{\alpha=1}^{3} \sum_{k=1}^{4} b_{k j}^{\alpha} y_{4 l+k} \frac{\partial s}{\partial x_{\alpha+1}}\left(|y|^{2}, t_{1}, t_{2}, t_{3}\right) \\
& =2 y_{4 l+j}\left[-c_{n-1} \sum_{k=0}^{2 n-1}(2 n-1) !(2 n-k)(k+1) \frac{\left(|y|^{2}-t\right)}{\left(|y|^{2}+|t| \mathbf{i}\right)^{2 n-k+1}\left(|y|^{2}-|t| \mathbf{i}\right)^{k+2}}\right.
\end{aligned}
$$




$$
\begin{gathered}
\left.+c_{n-1} \sum_{k=0}^{2 n-2}(2 n-1) !(2 n-k-1)(k+1) \frac{1}{\left(|y|^{2}+|t| \mathbf{i}\right)^{2 n-k}\left(|y|^{2}-|t| \mathbf{i}\right)^{k+2}}\right] \\
+2 \sum_{\alpha=1}^{3} \sum_{k=1}^{4} b_{k j}^{\alpha} y_{4 l+k}\left\{c_{n-1} \sum_{k=0}^{2 n-2}(2 n-2) !(2 n-k-1)(k+1)\right. \\
\quad\left[-\frac{1}{\left(|y|^{2}+|t| \mathbf{i}\right)^{2 n-k}\left(|y|^{2}-|t| \mathbf{i}\right)^{k+2}} \mathbf{i}_{\alpha}-\frac{(2 n-k)\left(|y|^{2}-t\right)}{\left(|y|^{2}+|t| \mathbf{i}\right)^{2 n-k+1}\left(|y|^{2}-|t| \mathbf{i}\right)^{k+2}} \frac{t_{\alpha}}{|t|} \mathbf{i}_{\alpha}\right. \\
\left.\quad+\frac{(k+2)\left(|y|^{2}-t\right)}{\left(|y|^{2}+|t| \mathbf{i}\right)^{2 n-k}\left(|y|^{2}-|t| \mathbf{i}\right)^{k+3}} \frac{t_{\alpha}}{|t|} \mathbf{i}_{\alpha}\right] \\
-c_{n-1} \sum_{k=0}^{2 n-3}(2 n-2) !(2 n-k-2)(k+1)\left[-\frac{(2 n-k-1)}{\left(|y|^{2}+|t| \mathbf{i}\right)^{2 n-k}\left(|y|^{2}-|t| \mathbf{i}\right)^{k+2}} \frac{t_{\alpha}}{|t|} \mathbf{i}_{\alpha}\right. \\
\left.+\frac{t_{\alpha}}{\left(|y|^{2}+|t| \mathbf{i}\right)^{2 n-k-1}\left(|y|^{2}-|t| \mathbf{i}\right)^{k+3}} \frac{\left.\left.\mathbf{i}_{\alpha}\right]\right\}}{|t|}\right]
\end{gathered}
$$

$=: I+I I$.

For the term $I$, by definition, we have

$$
\begin{aligned}
|I| \leq & 2 c_{n-1} \sum_{k=0}^{2 n-1}(2 n-1) !(2 n-k)(k+1) \frac{\left(|y|^{4}+|t|^{2}\right)^{\frac{1}{2}}\left|y_{4 l+j}\right|}{\left(|y|^{4}+|t|^{2}\right)^{\frac{2 n-k+1}{2}}\left(|y|^{4}+|t|^{2}\right)^{\frac{k+2}{2}}} \\
& +(2 n-1) c_{n-1} \sum_{k=0}^{2 n-2}(2 n-2) !(2 n-k-1)(k+1) \frac{\left|y_{4 l+j}\right|}{\left(|y|^{4}+|t|^{2}\right)^{\frac{2 n-k}{2}}\left(|y|^{4}+|t|^{2}\right)^{\frac{k+2}{2}}} \\
\leq & C \frac{1}{\left(|y|^{4}+|t|^{2}\right)^{n+\frac{3}{4}}} \\
= & C \frac{1}{\|g\|^{Q+1}} .
\end{aligned}
$$

For the term $I I$, we find

$$
\begin{aligned}
& |I I| \leq 2 \sum_{\alpha=1}^{3} \sum_{k=1}^{4 n-4}\left|b_{k j}^{\alpha}\right|\left|y_{4 l+k}\right| \\
& \times\left\{c _ { n - 1 } \sum _ { k = 0 } ^ { 2 n - 2 } ( 2 n - 2 ) ! ( 2 n - k - 1 ) ( k + 1 ) \left[\frac{1}{\left(|y|^{4}+|t|^{2}\right)^{\frac{2 n-k}{2}}\left(|y|^{4}+|t|^{2}\right)^{\frac{k+2}{2}}}\right.\right. \\
& \left.+\frac{(2 n-k)\left(|y|^{4}+|t|^{2}\right)^{\frac{1}{2}}}{\left(|y|^{4}+|t|^{2}\right)^{\frac{2 n-k+1}{2}}\left(|y|^{4}+|t|^{2}\right)^{\frac{k+2}{2}}} \frac{\left|t_{\alpha}\right|}{|t|}+\frac{(k+2)\left(|y|^{4}+|t|^{2}\right)^{\frac{1}{2}}}{\left(|y|^{4}+|t|^{2}\right)^{\frac{2 n-k}{2}}\left(|y|^{4}+|t|^{2}\right)^{\frac{k+3}{2}}} \frac{\left|t_{\alpha}\right|}{|t|}\right] \\
& +c_{n-1} \sum_{k=0}^{2 n-3}(2 n-2) !(2 n-k-2)(k+1)\left[\frac{(2 n-k-1)}{\left(|y|^{4}+|t|^{2}\right)^{\frac{2 n-k}{2}}\left(|y|^{4}+|t|^{2}\right)^{\frac{k+2}{2}}} \frac{\left|t_{\alpha}\right|}{|t|}\right. \\
& \left.\left.+\frac{k+2}{\left(|y|^{4}+|t|^{2}\right)^{\frac{2 n-k-1}{2}}\left(|y|^{4}+|t|^{2}\right)^{\frac{k+3}{2}}} \frac{\left|t_{\alpha}\right|}{|t|}\right]\right\} \\
& \leq 2 \sum_{\alpha=1}^{3} \sum_{k=1}^{4 n-4}\left|b_{k j}^{\alpha}\right|\left\{c_{n-1} \sum_{k=0}^{2 n-2}(2 n-2) !(2 n-k-1)(k+1)\right. \\
& \times\left[\frac{1}{\left(|y|^{4}+|t|^{2}\right)^{n+\frac{3}{4}}}+\frac{(2 n-k)}{\left(|y|^{4}+|t|^{2}\right)^{n+\frac{3}{4}}}+\frac{(k+2)}{\left(|y|^{4}+|t|^{2}\right)^{n+\frac{3}{4}}}\right]
\end{aligned}
$$




$$
\begin{aligned}
& \left.+c_{n-1} \sum_{k=0}^{2 n-3}(2 n-2) !(2 n-k-2)(k+1) \times\left[\frac{(2 n-k-1)}{\left(|y|^{4}+|t|^{2}\right)^{n+\frac{3}{4}}}+\frac{(2 n-k)}{\left(|y|^{4}+|t|^{2}\right)^{n+\frac{3}{4}}}\right]\right\} \\
\leq & C \frac{1}{\left(|y|^{4}+|t|^{2}\right)^{n+\frac{3}{4}}} \\
= & C \frac{1}{\|g\|^{Q+1}},
\end{aligned}
$$

which implies (1.6) holds.

Therefore, combining the estimates of $I$ and $I I$ we obtain that

$$
\left|Y_{4 l+j} K(g)\right| \leq C \frac{1}{\|g\|^{Q+1}} .
$$

Thus

$$
\left|Y_{4 l+j} K(x \cdot u)\right| \leq C \frac{1}{\|x \cdot u\|^{Q+1}}=C \frac{1}{\rho\left(u, x^{-1}\right)^{Q+1}} .
$$

Since $\rho$ is a quasi-distance, by (2.9), (3.5) and the fact that $\rho\left(g_{0}, h\right) \geq c \rho\left(g, g_{0}\right)$, we have

$$
\begin{aligned}
\rho\left(u, x^{-1}\right) & \geq \frac{1}{C_{\rho}} \rho\left(0, x^{-1}\right)-\rho(0, u) \geq \frac{1}{C_{\rho}} \rho\left(g_{0}, h\right)-\frac{\tilde{C}_{d}}{C_{d}} \rho\left(g, g_{0}\right) \\
& \geq \frac{1}{C_{\rho}} \rho\left(g_{0}, h\right)-\frac{\tilde{C}_{d}}{c C_{d}} \rho\left(g_{0}, h\right) \\
& =\left(\frac{1}{C_{\rho}}-\frac{\tilde{C}_{d}}{c C_{d}}\right) \rho\left(g_{0}, h\right) .
\end{aligned}
$$

Consequently,

$$
\left|Y_{4 l+j} K(x \cdot u)\right| \lesssim \frac{1}{\rho\left(g_{0}, h\right)^{Q+1}}
$$

Together with (3.7) we can see

$$
\left|K(g, h)-K\left(g_{0}, h\right)\right| \lesssim \frac{\rho\left(g, g_{0}\right)}{\rho\left(g_{0}, h\right)^{Q+1}} .
$$

Similarly, if $\rho\left(g, h_{0}\right) \geq c \rho\left(h, h_{0}\right)$, we can obtain

$$
\left|K(g, h)-K\left(g, h_{0}\right)\right| \lesssim \frac{\rho\left(h, h_{0}\right)}{\rho\left(g, h_{0}\right)^{Q+1}} .
$$

The proof of Theorem 1.2 is complete.

Proof of Theorem 1.4. We prove this theorem by 4 steps, using the idea in [14].

Step 1. We claim that: "there exists a point $g_{0}$ on the unit sphere of $\mathscr{H}^{n-1}$ such that $K\left(g_{0}\right) \neq 0 "$.

To see this, we choose $g_{0}=\left(\frac{\mathrm{i}}{\sqrt{2}}, \frac{y}{\sqrt[4]{2}}\right) \in \mathscr{H}^{n-1}$ such that $|y|=1$. It is clear that this $g_{0}$ is on the unit sphere of $\mathscr{H}^{n-1}$.

By (2.8), we have

$$
K\left(\delta_{\sqrt[4]{2}} g_{0}\right)=s(1+\mathbf{i})
$$

Based on the equality (3.3) in the proof of Theorem 1.2, we see that for $\sigma=1+\mathbf{i}$, we have $z=1+\mathbf{i}$ such that

$$
s(1+\mathbf{i})=c_{n-1} \frac{2(2 n-2) !}{|z|^{4}(z-\bar{z})^{3}}
$$




$$
\begin{aligned}
& \times\left\{\left[\frac{\bar{z}^{2}}{z^{2 n-2}}\left(z+(2 n-1) \frac{z-\bar{z}}{2}\right)-\frac{z^{2}}{\bar{z}^{2 n-2}}\left(\bar{z}-(2 n-1) \frac{z-\bar{z}}{2}\right)\right] \bar{\sigma}\right. \\
& \left.\quad-\left[\frac{\bar{z}^{2}}{z^{2 n-3}}\left(z+(2 n-2) \frac{z-\bar{z}}{2}\right)-\frac{z^{2}}{\bar{z}^{2 n-3}}\left(\bar{z}-(2 n-2) \frac{z-\bar{z}}{2}\right)\right]\right\} \\
& :=-c_{n-1} \frac{(2 n-2) !}{16 \mathbf{i}} \times A .
\end{aligned}
$$

For the term $A$, we further have

$$
\begin{aligned}
& A=\left[\frac{(1-\mathbf{i})^{2}}{(1+\mathbf{i})^{2 n-2}}(1+\mathbf{i}+(2 n-1) \mathbf{i})-\frac{(1+\mathbf{i})^{2}}{(1-\mathbf{i})^{2 n-2}}(1-\mathbf{i}-(2 n-1) \mathbf{i})\right](1-\mathbf{i}) \\
& -\left[\frac{(1-\mathbf{i})^{2}}{(1+\mathbf{i})^{2 n-3}}(1+\mathbf{i}+(2 n-2) \mathbf{i})-\frac{(1+\mathbf{i})^{2}}{(1-\mathbf{i})^{2 n-3}}((1-\mathbf{i})-(2 n-2) \mathbf{i})\right] \\
& =\frac{(1-\mathbf{i})^{3}}{(1+\mathbf{i})^{2 n-2}}(1+2 n \mathbf{i})-\frac{(1+\mathbf{i})^{2}}{(1-\mathbf{i})^{2 n-3}}(1-2 n \mathbf{i})-\frac{(1-\mathbf{i})^{2}}{(1+\mathbf{i})^{2 n-3}}(1+(2 n-1) \mathbf{i}) \\
& +\frac{(1+\mathbf{i})^{2}}{(1-\mathbf{i})^{2 n-3}}(1-(2 n-1) \mathbf{i}) \\
& =\frac{(1-\mathbf{i})^{3}(1+\mathbf{i})^{2}}{(1+\mathbf{i})^{2 n}}(1+2 n \mathbf{i})-\frac{(1-\mathbf{i})^{2}(1+\mathbf{i})^{3}}{(1+\mathbf{i})^{2 n}}(1+(2 n-1) \mathbf{i}) \\
& +\frac{(1+\mathbf{i})^{2}(1-\mathbf{i})^{3}}{(1-\mathbf{i})^{2 n}}[1-(2 n-1) \mathbf{i}-(1-2 n \mathbf{i})] \\
& =\frac{4}{(1+\mathbf{i})^{2 n}}[(1-\mathbf{i})(1+2 n \mathbf{i})-(1+\mathbf{i})(1+(2 n-1) \mathbf{i})]+\frac{4}{(1-\mathbf{i})^{2 n}}(1-\mathbf{i}) \mathbf{i} \\
& =\frac{4}{(1+\mathbf{i})^{2 n}}(4 n-1-\mathbf{i})+\frac{4}{(1-\mathbf{i})^{2 n}}(\mathbf{i}+1) \\
& =\frac{4}{(2 i)^{n}}\left[4 n-1-\mathbf{i}+(-1)^{n}(\mathbf{i}+1)\right] \neq 0 .
\end{aligned}
$$

Therefore,

$$
K\left(\delta_{\sqrt[4]{2}} g_{0}\right) \neq 0
$$

Then by Corollary 3.1, we see that $K\left(g_{0}\right) \neq 0$, which shows that the claim holds.

Step 2. We claim that: "there is a positive constant $C$ such that for every $R>0$ and every $g \in \mathscr{H}^{n-1}$, there exists a point $g_{*}$ in $\mathscr{H}^{n-1}$ such that $\rho\left(g, g_{*}\right)=R$ and that $\left|K\left(g, g_{*}\right)\right| \approx \frac{1}{R^{Q}} "$.

To see this, for every $R>0$ and every $g \in \mathscr{H}^{n-1}$, we set

$$
g_{*}=g \cdot \delta_{R}\left(g_{0}^{-1}\right)
$$

Then it is clear that

$$
\rho\left(g, g_{*}\right)=\rho\left(g, g \cdot \delta_{R}\left(g_{0}^{-1}\right)\right)=R \rho\left(\mathbf{0}, g_{0}^{-1}\right)=R \rho\left(\mathbf{0}, g_{0}\right)=R .
$$

Next, we note that from Corollary 3.1

$$
K\left(g, g_{*}\right)=K\left(g, g \cdot \delta_{R}\left(g_{0}^{-1}\right)\right)=K\left(\mathbf{0}, \delta_{R}\left(g_{0}^{-1}\right)\right)=R^{-Q} K\left(\mathbf{0}, g_{0}^{-1}\right)=R^{-Q} K\left(g_{0}\right) .
$$

Hence, we obtain that

$$
\left|K\left(g, g_{*}\right)\right|=R^{-Q}\left|K\left(g_{0}\right)\right|=: C_{0} R^{-Q}
$$

and since from Step 1 we know that $K\left(g_{0}\right)$ is non-zero. 
Step 3. We claim that: there exist positive constants $C$ and $R$ such that for every ball $B=B(g, r) \subset \mathscr{H}^{n-1}$ with $r>0$ and $g \in \mathscr{H}^{n-1}$, there exists another ball $\tilde{B}=B\left(g_{*}, r\right)$ in $\mathscr{H}^{n-1}$ such that $\rho\left(g, g_{*}\right)=R r$ and that for every $g_{1} \in B$ and $g_{2} \in \tilde{B}$,

$$
\left|K\left(g_{1}, g_{2}\right)\right| \geq \frac{C}{\rho\left(g_{1}, g_{2}\right)^{Q}} .
$$

To see this, we first note that $K$ satisfies Hölder's regularity. Hence, for the point $g_{0}$ obtained in Step 1, there exists a small positive constant $\epsilon_{0}<1$ such that

$$
K(\tilde{g}) \neq 0 \quad \text { and } \quad|K(\tilde{g})|>\frac{1}{2}\left|K\left(g_{0}\right)\right|
$$

for all $\tilde{g} \in B\left(g_{0}, 2 C_{\rho} \epsilon_{0}\right)$.

Now for every $B=B(g, r) \subset \mathscr{H}^{n-1}$ with $r>0$ and $g \in \mathscr{H}^{n-1}$, by using the argument in Step 2 we see that there exists a point $g_{*}=g \cdot \delta_{R r}\left(g_{0}^{-1}\right)$ in $\mathscr{H}^{n-1}$ such that $\rho\left(g, g_{*}\right)=R r$ and that $\left|K\left(g, g_{*}\right)\right| \approx \frac{1}{(R r)^{Q}}$, where $R$ is chosen to be large enough such that $\frac{1}{2 \epsilon_{0}}<R<\frac{1}{\epsilon_{0}}$.

Then for every $g_{1} \in B$ there exists $g_{1}^{\prime} \in B\left(\mathbf{0}, \epsilon_{0}\right)$ such that we can write $g_{1}=g \cdot \delta_{R r}\left(g_{1}^{\prime}\right)$. Also, for every $g_{2} \in B\left(g_{*}, r\right)$, there exists $g_{2}^{\prime} \in B\left(\mathbf{0}, \epsilon_{0}\right)$ such that we can write $g_{2}=$ $g_{*} \cdot \delta_{R r}\left(g_{2}^{\prime}\right)$. Now we have

$$
\begin{aligned}
K\left(g_{1}, g_{2}\right) & =K\left(g \cdot \delta_{R r}\left(g_{1}^{\prime}\right), g_{*} \cdot \delta_{R r}\left(g_{2}^{\prime}\right)\right)=K\left(g \cdot \delta_{R r}\left(g_{1}^{\prime}\right), g \cdot \delta_{R r}\left(g_{0}^{-1}\right) \cdot \delta_{R r}\left(g_{2}^{\prime}\right)\right) \\
& =K\left(\delta_{R r}\left(g_{1}^{\prime}\right), \delta_{R r}\left(g_{0}^{-1}\right) \cdot \delta_{R r}\left(g_{2}^{\prime}\right)\right)=K\left(\delta_{R r}\left(g_{1}^{\prime}\right), \delta_{R r}\left(g_{0}^{-1} \cdot g_{2}^{\prime}\right)\right) \\
& =(R r)^{-Q} K\left(g_{1}^{\prime}, g_{0}^{-1} \cdot g_{2}^{\prime}\right) \\
& =(R r)^{-Q} K\left(\left(g_{2}^{\prime}\right)^{-1} \cdot g_{0} \cdot g_{1}^{\prime}\right) .
\end{aligned}
$$

Next, note that

$$
\begin{aligned}
\rho\left(\left(g_{2}^{\prime}\right)^{-1} \cdot g_{0} \cdot g_{1}^{\prime}, g_{0}\right) & =\rho\left(g_{0} \cdot g_{1}^{\prime}, g_{2}^{\prime} \cdot g_{0}\right) \leq C_{\rho}\left[\rho\left(g_{0} \cdot g_{1}^{\prime}, g_{0}\right)+\rho\left(g_{0}, g_{2}^{\prime} \cdot g_{0}\right)\right] \\
& \leq C_{\rho}\left[\rho\left(g_{1}^{\prime}, 0\right)+\rho\left(0, g_{2}^{\prime}\right)\right] \leq 2 C_{\rho} \epsilon_{0},
\end{aligned}
$$

which shows that $\left(g_{2}^{\prime}\right)^{-1} \cdot g_{0} \cdot g_{1}^{\prime} \in B\left(g_{0}, 2 C_{\rho} \epsilon_{0}\right)$. Then by (3.8), we have

$$
\left|K\left(\left(g_{2}^{\prime}\right)^{-1} \cdot g_{0} \cdot g_{1}^{\prime}\right)\right|>\frac{1}{2}\left|K\left(g_{0}\right)\right| \text {. }
$$

Therefore,

$$
K\left(g_{1}, g_{2}\right)>\frac{1}{2(R r)^{Q}}\left|K\left(g_{0}\right)\right| \geq C\left(K, C_{\rho}\right) \frac{1}{\rho\left(g_{1}, g_{2}\right)^{Q}},
$$

for any $g_{1} \in B(g, r)$ and any $g_{2} \in B\left(g_{*}, r\right)$.

Step 4. Since $K$ is continuous on $\mathscr{H}^{n-1} \backslash\{\mathbf{0}\}$, by Step $\mathbf{1}$. we can see that there exists a compact set $\Omega$ on the unit sphere $\mathcal{S}(\mathbf{0}, 1)$ of $\mathscr{H}^{n-1}$ with $m(\Omega)>0$, where $m$ is the Radon measure on $\mathcal{S}(\mathbf{0}, 1)$, such that $K(g) \neq 0$ for any $g \in \Omega$. Then by the similar proof to that of [5. Theorem 1.1], we can find a positive constant $r_{0}$ and a "twisted truncated sector" $S_{g} \subset \mathscr{H}^{n-1}$ such that

$$
\inf _{g^{\prime} \in S_{g}} \rho\left(g, g^{\prime}\right)=r_{0}
$$

and that for every $g_{1} \in B(g, 1)$ and $g_{2} \in S_{g}$ we have

$$
\left|K\left(g_{1}, g_{2}\right)\right| \geq \frac{C\left(K, C_{\rho}\right)}{\rho\left(g_{1}, g_{2}\right)^{Q}} .
$$

Moreover, this sector $S_{g}$ is regular in the sense that $\left|S_{g}\right|=\infty$ and that for every $R_{2}>R_{1}>$ $2 r_{0}$

$$
\left|\left(B\left(g, R_{2}\right) \backslash B\left(g, R_{1}\right)\right) \cap S_{g}\right| \approx\left|B\left(g, R_{2}\right) \backslash B\left(g, R_{1}\right)\right|
$$

with the implicit constants independent of $g$ and $R_{1}, R_{2}$. The proof of Theorem 1.4 is complete. 
Remark 3.3. From the proof of Theorem 1.4, we see that we have the following version of kernel lower bound concerning the real coordinate of each component.

Since $K(g, h)$ is $\mathbb{H}$-valued, we write

$$
K(g, h)=K_{1}(g, h)+K_{2}(g, h) \mathbf{i}+K_{3}(g, h) \mathbf{j}+K_{4}(g, h) \mathbf{k},
$$

where each $K_{i}(g, h)$ is real-valued, $i=1,2,3,4$.

Then following kernel lower bound in Theorem 1.4 and using the idea of Hytönen [19] we see that at least one of the $K_{i}$ above satisfies the following argument:

There exist positive constants $3 \leq A_{1} \leq A_{2}$ such that for any ball $B:=B\left(g_{0}, r\right) \subset \mathscr{H}^{n-1}$, there exist another ball $\widetilde{B}:=B\left(h_{0}, r\right) \subset \mathscr{H}^{n-1}$ such that $A_{1} r \leq d\left(g_{0}, h_{0}\right) \leq A_{2} r$, and for all $(g, h) \in(B \times \widetilde{B}), K(g, h)$ does not change sign and

$$
|K(g, h)| \geq \frac{C}{\rho(g, h)^{Q}} .
$$

Proof of Theorem 1.5. The argument (i) follows from the kernel lower bound obtained in Theorem [1.4 and the standard proof in [19] (also can be achieved by following [11).

It suffices to prove the "only if" part of (ii), since the "if" part follows from the argument in 5 .

To see this, we seek to get a contradiction by using the idea (due to M. Lacey) stated at the beginning of proof of (2) of Theorem 1.1 in [13], that is, in its simplest form, there is no bounded operator $T: \ell^{p}(\mathbb{N}) \rightarrow \ell^{p}(\mathbb{N})$ with $T e_{j}=T e_{k} \neq 0$ for all $j, k \in \mathbb{N}$. Here, $e_{j}$ is the standard basis for $\ell^{p}(\mathbb{N})$.

To get to this contradiction, we just need to use the kernel lower bound in (3.9), and then combine the argument used in the proof of (2) of Theorem 1.1 in [13]. Repeating the process there almost step by step, we obtain the "only if" part. We leave the details to readers.

Acknowledgement: Chang is supported by NSF grant DMS-1408839 and a McDevitt Endowment Fund at Georgetown University. Duong and Li are supported by the Australian Research Council (ARC) through the research grants DP170101060 and DP190100970 and by Macquarie University Research Seeding Grant. Wang is supported by National Nature Science Foundation in China (No. 11571305). Wu is supported by NSF of China (Grants No. 11671185 and No. 11701250), the Natural Science Foundation of Shandong Province (No. ZR2018LA002 and No. ZR2019YQ04).

\section{REFERENCES}

1. S. Alesker, Non-commmutative linear algebra and plurisubharmonic functions of quaternionic variables, Bull. Sci. Math., 127 (2003), no. 1, 1-35.

2. A. Bonfiglioli, E. Lanconelli and F. Uguzzoni, Stratied Lie groups and potential theory for their subLaplacians, Springer Monographs in Mathematics, Springer-Verlag, 2007.

3. D.-C. Chang and I. Markina, Quaternion $H$-type group and differential operator $\Delta_{\lambda}$, Sci. China Ser. A, 51 (2008), no. 4, 523-540.

4. D.-C. Chang, I. Markina and W. Wang, On the Cauchy-Szegö kernel for quaternion Siegel upper halfspace, Complex Anal. Oper. Theory, 7 (2013), no. 5, 1623-1654.

5. P. Chen, X.T. Duong, J. Li and Q.Y. Wu, Compactness of Riesz transform commutator on stratified Lie groups, J. Funct. Anal., 277 (2019), no. 6, 1639-1676.

6. W.L. Chow, Über Systeme von linearen partiellen differentialgleichungen erster ordnung (in German), Math. Ann., 117 (1939), 98-105.

7. M. Christ, H. Liu and A. Zhang, Sharp Hardy-Littlewood-Sobolev inequalities on quaternionic Heisenberg groups, Nonlinear Anal., 130 (2016), 361-395.

8. R. Coifman, R. Rochberg and G. Weiss, Factorization theorems for Hardy spaces in several variables, Ann. of Math. (2), 103 (1976), 611-635.

9. F. Colombo, I. Sabadini, F. Sommen and D. Struppa, Analysis of Dirac systems and computational algebra, Progress in Mathematical Physics 39, Boston, Birkhäuser, 2004. 
10. G.B. Folland and E.M. Stein, Hardy spaces on homogeneous groups, in: Mathematical Notes, vol. 28, Princeton University Press, University of Tokyo Press, Princeton N.J., Tokyo, 1982.

11. X.T. Duong, R. Gong, M.-J. S. Kuffner, J. Li, B.D. Wick and D.Y. Yang, Two weight commutators on spaces of homogeneous type and applications, arXiv:1809.07942.

12. X.T. Duong, I. Holmes, J. Li, B.D. Wick and D.Y. Yang, Two weight Commutators in the Dirichlet and Neumann Laplacian settings, J. Funct. Anal., 276 (2019), 1007-1060.

13. X.T. Duong, M. Lacey, J. Li, B.D. Wick and Q.Y. Wu, Commutators of Cauchy type integrals for domains in $\mathbb{C}^{n}$ with minimal smoothness, to appear in Indiana Univ. Math. J.

14. X.T. Duong, H.-Q. Li, J. Li and B.D. Wick, Lower bound for Riesz transform kernels and commutator theorems on stratified nilpotent Lie groups, J. Math. Pures Appl., (9) 124 (2019), 273-299.

15. X.T. Duong, J. Li, B.D. Wick and D.Y. Yang, Factorization for Hardy spaces and characterization for BMO spaces via commutators in the Bessel setting, Indiana Univ. Math. J., 66 (2017), no. 4, 1081-1106.

16. W.C. Guo, J.L. Lian and H.X. Wu, The unified theory for the necessity of bounded commutators and applications, to appear in J. Geom. Anal.

17. W.C. Guo, H.X. Wu and D.Y. Yang, A revisit on the compactness of commutators, arXiv:1712.08292.

18. I. Holmes, M. Lacey and B.D. Wick, Commutators in the two-weight setting, Math. Ann., 367 (2017), $51-80$.

19. T. Hytönen, The $L^{p}$ to $L^{q}$ boundedness of commutators with applications to the Jacobian operator, arXiv:1804.11167.

20. S. Ivanov, I. Minchev and D. Vassilev, Quaternionic contact Einstein structures and the quaternionic contact Yamabe problem, Memoirs of the American Mathematical Society, vol. 231, (2014) no. 1086, pp. vi+82.

21. A. Korányi and H.M. Reimann, Quasiconformal mappings on the Heisenberg group, Invent. Math., 80 (1985), 309-338.

22. A. Korányi and H.M. Reimann, Foundations for the theory of quasiconformal mappings on the Heisenberg group, Adv. Math., 111 (1995), no. 1, 1-87.

23. A.K. Lerner, S. Ombrosi and I.P. Rivera-Ríos, On pointwise and weighted estimates for commutators of Calderón-Zygmund operators. Adv. Math., 319 (2017), 153-181.

24. A.K. Lerner, S. Ombrosi and I.P. Rivera-Ríos, Commutators of singular integrals revisited, Bull. Lond. Math. Soc., 51 (2019), no. 1, 107-119.

25. J. Li, T. Nguyen, L.A. Ward and B.D. Wick, The Cauchy integral, bounded and compact commutators, to appear in Studia Math.

26. J. Li and B.D. Wick, Characterizations of $H_{\Delta_{N}}^{1}\left(\mathbb{R}^{n}\right)$ and $\mathrm{BMO}_{\Delta_{N}}\left(\mathbb{R}^{n}\right)$ via Weak Factorizations and Commutators, J. Funct. Anal., 272 (2017), 5384-5416.

27. P. Pansu, Métriques de Carnot-Carathéodory et quasiisométries des espaces symétriques de rang un, Ann. of Math., 129 (1989), no. 1, 1-60.

28. Y. Shi and W. Wang, On conformal qc geometry, spherical qc manifolds and convex cocompact subgroups of $\operatorname{Sp}(n+1,1)$, Ann. Global Anal. Geom., 49 (2016), no. 3, 271-307.

29. Y. Shi and W. Wang, The Szegö kernel for $k$-CF functions on the quaternionic Heisenberg group, Appl. Anal., 14 (2017), 2474-2492.

30. E.M. Stein, Harmonic Analysis Real-Variable Methods, Orthogonality, and Oscillatory Integrals, Princeton Mathematical Series, 43, Princeton University Press, Princeton, New Jersey, 1993.

31. J. Tao, D.C. Yang and D.Y. Yang, Boundedness and compactness characterizations of Cauchy integral commutators on Morrey spaces, Math. Meth. Appl. Sci., 42 (2019), 1631-1651.

32. A. Uchiyama, On the compactness of operators of Hankel type, Tôhoku Math. J., (2) 30 (1978), no. 1, $163-171$.

33. D. Wan and W. Wang, On quaternionic Monge-Ampère operator, closed positive currents and LelongJensen type formula on the quaternionic space, Bull. Sci. Math., 141 (2017), 267-311.

34. W. Wang, The tangential Cauchy-Fueter complex on the quaternionic Heisenberg group, J. Geom. Phys., 61 (2011), 363-380.

35 . W. Wang, The Neumann problem for the $k$-Cauchy-Fueter complex over $k$-pseudoconvex domains in $\mathbb{R}^{4}$ and the $L^{2}$ estimate, J. Geom. Anal., 29 (2019), 1233-1258.

Der-Chen Chang, Department of Mathematics and Department of Computer Science, Georgetown University, Washington D.C. 20057, USA and Graduate Institute of Business Administration, College of Management, Fu Jen Catholic University, New Taipei City 242, Taiwan, ROC.

E-mail address: chang@georgetown.edu 
Xuan Thinh Duong, Department of Mathematics, Macquarie University, NSW, 2109, AusTRALIA

E-mail address: xuan.duong@mq.edu.au

Ji Li, Department of Mathematics, Macquarie University, NSW, 2109, Australia

E-mail address: ji.li@mq.edu.au

Wei Wang, Department of Mathematics, Zhejiang University, Zhejiang 310027, China

E-mail address: wwang@zju.edu.cn

Qingyan Wu, Department of Mathematics, Linyi University, Shandong, 276005, China

E-mail address: qingyanwu@gmail.com 